INPLASY

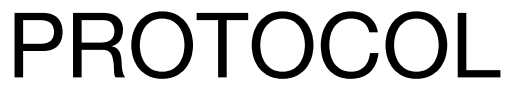

To cite: Cheng et al. Efficiency of red cell distribution width in predicting severity and mortality of patients with acute pancreatitis: A protocol for systematic review and metaanalysis. Inplasy protocol 202110042. doi:

10.37766/inplasy2021.1.0042

Received: 14 January 2021

Published: 14 January 2021

Corresponding author: Tao Cheng

liubofubeau@163.com

Author Affiliation:

Emergency Department, West China Hospital

Support: None.

Review Stage at time of this submission: The review has not yet started.

Conflicts of interest:

None.

\section{Efficiency of red cell distribution width in predicting severity and mortality of patients with acute pancreatitis: A protocol for} systematic review and meta-analysis

Cheng, T1; Liu, B2; Liu, J3; He, Y4; Pan, P5; Lai, Q6; Han, T7; Cao, ${ }^{8}$.

Review question / Objective: The objective of the study was to investigate if red cell distribution width can predict the severity and mortality of patients with acute pancreatitis accurately at an early stage.

Condition being studied: Acute pancreatitis (AP) is a sudden inflammatory process in the pancreas with variable involvement of nearby organs or other organ systems. And it is a common cause for hospitalization of gastrointestinal origin. Predicting the severity of AP early is important to help us triage and decrease mortality. Red cell distribution width (RDW) is reflective of systemic inflammation. The objective of the study was to investigate if RDW can predict the severity and mortality of AP accurately at an early stage.

INPLASY registration number: This protocol was registered with the International Platform of Registered Systematic Review and Meta-Analysis Protocols (INPLASY) on 14 January 2021 and was last updated on 14 January 2021 (registration number INPLASY202110042).

\section{INTRODUCTION}

Review question / Objective: The objective of the study was to investigate if red cell distribution width can predict the severity and mortality of patients with acute pancreatitis accurately at an early stage.
Condition being studied: Acute pancreatitis (AP) is a sudden inflammatory process in the pancreas with variable involvement of nearby organs or other organ systems. And it is a common cause for hospitalization of gastrointestinal origin. Predicting the severity of AP early is important to help us 
triage and decrease mortality. Red cell distribution width (RDW) is reflective of systemic inflammation. The objective of the study was to investigate if RDW can predict the severity and mortality of AP accurately at an early stage.

\section{METHODS}

Participant or population: Inclusion criteria were age ?18 years old, a minimum hospital stay of $24 \mathrm{~h}$ and a diagnosis of AP? according to the 2012 revision of the Atlanta classification and definitions by the international consensus?

Intervention: Red cell distribution width (RDW) is a routine parameter of the complete blood count test, described as simple, easy, inexpensive and quantitative that measures the size heterogeneity of peripheral red blood cell (RBC), known as anisocytosis. Several studies have shown that RDW may be an inflammatory status, such as C-reactive protein, interleukin 6, and fibrinogen, and it may be used to predict severity and mortality in patients with AP. To identify whether RDW is a simple, easy, and sensitive marker to predict the severity and mortality of AP, we conducted this meta-analysis.

Comparator: Severity and mortality of AP patients with high red cell distribution width vs. severity and mortality of AP patients with low red cell distribution width.

Study designs to be included: No restrictions on the types of study design.

Eligibility criteria: Exclusion criteria included: age $<18$ years old, patients with chronic pancreatitis or pancreas carcinoma and patients with incomplete data and the presence of underlying factors that could change RDW, such as infectious or immunosuppressive conditions/therapy, active malignancy, late stage of liver cirrhosis, active tuberculosis, refractory heart failure, chronic use of erythropoietin, recent transfusion history, pregnancy or trauma.
Information sources: EMBASE, Web of Knowledge, PubMed, ClinicalTrials.gov and Cochrane Library were searched from October 25, 2012 to April 4, 2020.

Main outcome(s): 1.severity: MAP, MSAP, and SAP(according to the 2012 revision of the Atlanta classification and definitions by the international consensus?) or AP patients with APACHE II?8 and AP patients with APACHE II?8 2.mortality: hospital mortality or early mortality(?100days).

Data management: Important information included the name of the first author, publication year, publication country, type of study, study population, sample size, defined criteria of SAP, outcomes studied (severity and mortality rate) and the RDW.

Quality assessment / Risk of bias analysis: Risk of bias assessment was carried out according to the Newcastle-Ottawa Scale (NOS) to rate the internal validity of the individual studies, and funnel plots were constructed to assess the risk of publication bias.Two reviewers independently employed the searching strategy, with reading the papers and scoring them according to the QUADAS2 checklist; disagreement was settled by a third opinion. Important information was abstracted from the included articles in a standardized form by two reviewers.

Strategy of data synthesis: RDW of MAP patients vs. RDW of non-MAP patients, RDW of non-SAP patients vs. RDW of SAP patients, RDW of AP patients with APACHE II ?8 vs. RDW of AP patients with APACHE II ?8 and RDW of survival AP patients vs. RDW of non-survival AP patients (Inverse Variance random-effect method). Heterogeneity will be examined by computing the $Q$ statistic and $I^{2}$ statistic, and presence of reporting bias by visual inspection of funnel plots. All pairwise meta-analytic calculations were performed with Review Manager software (RevMan) version 5.3 (Cochrane Collaboration).

Subgroup analysis: RDW of MAP patients vs. RDW of non-MAP patients, RDW of nonSAP patients vs. RDW of SAP patients, 
RDW of AP patients with APACHE II ?8 vs. RDW of AP patients with APACHE II ?8 and RDW of survival AP patients vs. RDW of non-survival AP patients.

Sensibility analysis: The sensibility analysis will be performed if necessary.

Country(ies) involved: China.

Keywords: Acute pancreatitis, red cell distribution width, meta-analysis.

Contributions of each author:

Author 1 - Tao Cheng.

Author 2 - Bo-fu, Liu.

Author 3 - Jun-zhao Liu.

Author 4 - Ya-rong He.

Author 5 - Pan Pan.

Author 6 - Qiang Lai.

Author 7 - Tian-yong Han.

Author 8 - Yu Cao. 